\title{
Low-temperature entropy in Cu-based shape-memory alloys and the boson peak
}

\author{
Marcelo Stipcich,* Jordi Marcos, Lluís Mañosa, and Antoni Planes \\ Departament d'Estructura i Constituents de la Materia, Facultat de Física, Universitat de Barcelona, Diagonal 647, \\ E-08028 Barcelona, Catalonia, Spain \\ Ricardo Romero \\ IFIMAT, Universidad del Centro de la Provincia de Buenos Aires and Comisión de Investigaciones Científicas de la Provincia \\ de Buenos Aires, Pinto 399, 7000 Tandil, Argentina \\ (Received 13 August 2003; published 16 December 2003)
}

\begin{abstract}
We have measured the energy and entropy differences between $\beta$ and martensitic phases in $\mathrm{Cu}-\mathrm{Zn}$ and $\mathrm{Cu}-\mathrm{Zn}$-Al shape-memory alloy systems which display martensitic transitions in a range of temperatures between $30 \mathrm{~K}$ and $130 \mathrm{~K}$. We show that the entropy difference originates in the low-energy $T A_{2}$ vibrational modes and displays the predicted features for a system showing the so-called boson peak, characteristic of structurally disordered solids. In the studied systems the boson peak is conveniently revealed by the ratio between the entropy difference and the Debye entropy.
\end{abstract}

DOI: 10.1103/PhysRevB.68.214302

PACS number(s): 63.50.+x, 65.40.Gr, 81.30.Kf

\section{INTRODUCTION}

$\mathrm{Cu}$-based shape-memory alloys display an (open) bcc phase or $\beta$ phase at high temperature, and transform martensitically towards a close-packed structure at a lower temperature. For a considerable time, ${ }^{1}$ it has been recognized that the stability of the high-temperature open phase is a consequence of its excess of vibrational entropy compared with its close-packed counterpart, which is more favorable from purely energy considerations. ${ }^{2}$ This excess of entropy originates from low restoring forces in specific lattice directions related to the path that leads the system from the hightemperature parent phase to the martensite (combination of shears and shuffles on $\{110\}$ planes along $\langle 1 \overline{1} 0\rangle$ directions). Such low restoring forces manifest themselves by a lowenergy $T A_{2}[110]$ phonon branch with a correspondingly small elastic constant $C^{\prime}$, which slightly softens as the martensitic transition is approached. In contrast, in the martensitic phase, the boundary frequency of the $T A_{1}[100]$ branch $^{3}$ is three times larger than the corresponding boundary frequency of the $T A_{2}$ branch in the $\beta$ phase. At the same time the elastic anisotropy $C_{44} / C^{\prime}$ decreases from $\sim 13$ to $\sim 2 .^{4,5}$ This peculiar vibrational behavior shares some similarities with that of a number of structurally disordered solids. Actually, these systems also show an excess of low-frequency states not present in their crystalline counterpart. ${ }^{6,7}$ This fact suggests that the open $\beta$ phase can be considered, from the point of view of its vibrational properties, as a vibrationally disordered phase, and the martensitic transition as a condensation, with a first-order character, towards a vibrationally ordered phase. In disordered systems the excess of lowfrequency states is usually seen by a higher contribution compared with the Debye behavior which, in principle, is supposed to apply in the low-frequency regime. This excess of low-frequency states gives rise to the so-called boson peak (BP), which is shown by a maximum in the vibrational density of states (DOS) divided by $\omega^{2}$ at frequencies far below the Debye frequency (without a peak in the DOS itself). The
$\mathrm{BP}$ is also revealed by thermodynamic quantities. For instance, the heat capacity $C$ shows a peak in the scaled $C / T^{3}$ vs $T$ curve.

In the present paper we report measurements of the difference of enthalpy and entropy between $\beta$ and martensitic phases in two shape-memory alloy systems: $\mathrm{Cu}-\mathrm{Zn}$ and $\mathrm{Cu}-$ $\mathrm{Zn}$-Al. In the ternary alloy the compositions are selected with a constant valence electron to atom ratio $e / a$. The martensitic structures of these two alloy families are $9 R$ and $18 R$, respectively, which are monoclinic structures, and are conveniently described by a large unit cell (approximately orthorhombic) containing, 9 and 18 close-packed atomic planes, ${ }^{8}$ respectively. The distinct packing sequence is a consequence of the different ordering configurations in the two systems. $\mathrm{Cu}-\mathrm{Zn}$ displays a $\mathrm{B} 2(\operatorname{Im} 3 \mathrm{~m})$ ordered structure (nearest-neighbor ordering), while $\mathrm{Cu}-\mathrm{Zn}-\mathrm{Al}$ is $\mathrm{L}_{1}$ $(F m 3 m)$ (next nearest-neighbor ordering). It is worth mentioning that measurements of enthalpy and entropy changes have been previously reported for a number of $\mathrm{Cu}$-based shape-memory alloys, including those studied in this work. However, all previous data correspond to alloy systems transforming at relatively high temperatures. For this temperature range, $\Delta S$ has reached, within experimental error, the classical high-temperature value, and thus it does not show any appreciable temperature dependence. In the present paper measurements have been extended to systems transforming at relatively low temperatures. A simple vibrational model based on the ideas described above has been used in order to show that the entropy difference accounts fairly well for the excess of vibrational entropy of the high-temperature $\beta$ phase. Moreover, these results corroborate the existence of the BP in the studied shape-memory alloys.

\section{DENSITY OF STATES AND THE BOSON PEAK}

\section{A. General features}

We have collected reported DOS for $\mathrm{Cu}-\mathrm{Zn},{ }^{9} \mathrm{Cu}-\mathrm{Al}-\mathrm{Be},{ }^{10}$ and $\mathrm{Cu}-\mathrm{Al}-\mathrm{Mn}$ (Ref. 11) $\beta \mathrm{Cu}$-based alloys. From these data the ratio $g(\omega) / g_{D}(\omega)$ has been calculated, where $g(\omega)$ is the 


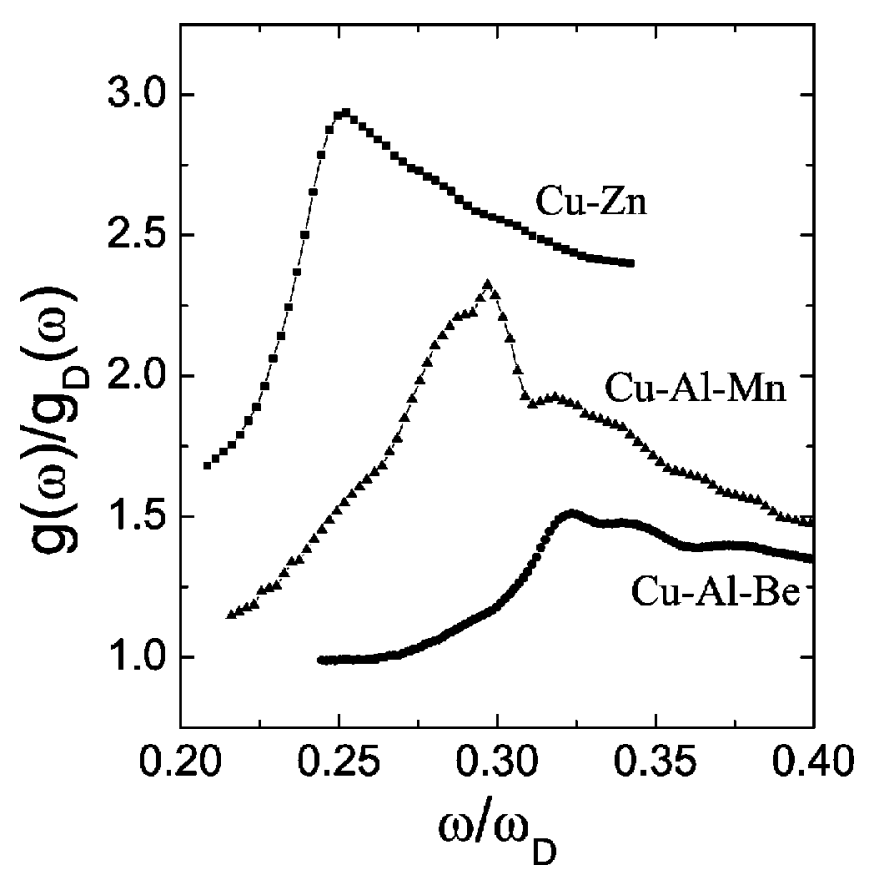

FIG. 1. $g(\omega) / g_{D}(\omega)$ vs $\omega / \omega_{D}$ for $\mathrm{Cu} ; 47$ at. $\% \mathrm{Zn}, \mathrm{Cu} ; 22.72$ at. $\% \mathrm{Al} ; 3.55$ at. $\% \mathrm{Be}$ and $\mathrm{Cu} ; 25$ at. $\% \mathrm{Al} ; 7$ at $\% \mathrm{Mn}$. The peak reveals the existence of a $\mathrm{BP}$ in these systems.

actual DOS and $g_{D}(\omega)$ is the Debye DOS $\left[g_{D}(\omega) \sim \omega^{2}\right]$. The ratio is shown in Fig. 1 as a function of $\omega / \omega_{D}\left(\omega_{D}\right.$ is the Debye frequency given in the corresponding references) for the three systems. A bump is clearly observed in the region of small frequencies $\left(\sim 0.25-0.35 \omega_{D}\right)$. It is worth noting that this bump is consistent with the flat $T A_{2}$ dispersion relation over a broad region of the Brillouin zone, and with corresponding low zone-boundary frequency which is close to the frequency of the maximum of the bump. This behavior is common to all Cu-based shape-memory alloys. These $T A_{2}$ anomalous modes correspond to displacements of $\{110\}$ planes along $\langle 1 \overline{1} 0\rangle$ direction, which are involved in the transformation from the $\beta$ phase to the close-packed martensitic structure. The behavior illustrated in Fig. 1, suggests that at low frequencies the DOS for shape-memory alloys can be expressed as

$$
g(\omega) \simeq A \omega^{2}+f\left(\omega-\omega_{E}\right),
$$

where the term $\omega^{2}$ describes $3 d$ Debye phonons, and the term $f\left(\omega-\omega_{E}\right)$ corresponds to a narrow distribution of phonons centered about $\omega_{E}$ and related to the anomalous phonon modes. The effect of the excess of low-frequency modes on the thermodynamic properties can be accounted for by simply assuming that $f\left(\omega-\omega_{E}\right)$ is a $\delta$ function. This means that the low-frequency transverse acoustic modes are essentially described in terms of an Einstein model with characteristic frequency $\omega_{E}$. While this is certainly too crude an approximation for the DOS, it is expected to provide reasonable results in terms of integral quantities. Thus, the following vibrational spectrum is assumed:

$$
g(\omega)= \begin{cases}9 N(1-p) \frac{\omega^{2}}{\omega_{D}^{3}}+3 N p \delta\left(\omega-\omega_{E}\right) & \text { for } \quad \omega \leqslant \omega_{D} \\ 0 & \text { for } \omega \geqslant \omega_{D},\end{cases}
$$

where $p$ is the fraction of anomalous or Einstein modes. The heat capacity of the $\beta$ phase is thus given by

$$
C_{\beta}(T)=(1-p) D\left(T / \theta_{D}\right)+p E\left(T / \theta_{E}\right),
$$

where $D\left(T / \theta_{D}\right)$ and $E\left(T / \theta_{E}\right)$ are Debye and Einstein heat capacities, respectively $\left(\theta_{D}=\hbar \omega_{D} / k\right.$ is the Debye temperature and $\theta_{E}=\hbar \omega_{E} / k$ is the Einstein temperature). The BP is revealed by a peak on the ratio $R_{C}=C_{\beta}(T) / D\left(T / \theta_{D}\right)$. Within the present model this ratio is given by

$$
R_{C}=1+p\left\{\frac{E\left(T / \theta_{E}\right)}{D\left(T / \theta_{D}\right)}-1\right\} .
$$

It is straightforward to show that for $T \rightarrow 0, R_{C} \rightarrow 1-p$; for $T \rightarrow \infty, R_{C} \rightarrow 1$, and that $R_{C}$ displays a maximum at a temperature $T_{\max }=\theta_{E} / 5$. Therefore, the proposed approach is adequate to model systems displaying the BP.

\section{B. The martensitic transition}

The martensitic phase is close packed and its vibrational properties can be adequately accounted for by a Debye model. Moreover, we will assume that the corresponding Debye temperature is the same as the Debye temperature of the $\beta$ phase. This means that the heat capacity of the martensitic phase is $C_{M}(T)=D\left(T / \theta_{D}\right)$. Within this framework, the modes responsible for the BP provide the excess of entropy of the $\beta$ phase with respect to the martensitic phase. Therefore, this excess of entropy for a system that transforms at a temperature $T_{M}$ is given by

$$
\Delta S=\int_{0}^{T_{M}} \frac{C_{\beta}-C_{M}}{T} d T=\int_{0}^{T_{M}}\left(R_{C}-1\right) \frac{D}{T} d T
$$

where $\Delta S=S_{\beta}-S_{M}$. Note that the BP also shows up in the ratio $\Delta S / S_{D}$ given by:

$$
\frac{\Delta S}{S_{D}}=\frac{\int_{0}^{T_{M}}\left(R_{C}-1\right) \frac{D}{T} d T}{\int_{0}^{T_{M} D} \frac{D}{T} d T} \equiv R_{S}-1
$$

The classical high-temperature limit of $\Delta S$ is given by

$$
\Delta S\left(T \gg \theta_{E}\right) \simeq 3 p N k \ln \frac{\theta_{D}}{\theta_{E}} .
$$

From this equation it is clear that the stability of the hightemperature $\beta$ phase is, for a given fraction $p$, favored by a low value of $\theta_{E}$. On the other hand, the high temperature mean-square displacement of $T A_{2}(110)[1 \overline{1} 0]$ Einstein modes is given by 


$$
\left\langle u_{T A_{2}}^{2}\right\rangle \simeq \frac{\hbar^{2} T}{k \theta_{E}^{2} M},
$$

where $M$ is the average atomic mass. Indeed, large-amplitude $T A_{2}(110)[1 \overline{1} 0]$ displacements enhances the instability towards the martensitic phase. Therefore, low $\theta_{E}$ also favors higher transition temperatures. From this point of view $T_{M}$ is the temperature at which both, the entropy stabilizing effect (thermodynamical) and the large-amplitude displacements destabilizing effect (mechanical) balance each other. This balance leads to the following relationship,

$$
T_{M} \simeq\left\langle u_{T A_{2}}^{2}\right\rangle \frac{M \omega_{D}^{2}}{k} e^{-2 \Delta S / 3 p N k} .
$$

Note that this expression only applies at temperatures $T$ $\gg \theta_{E}$.

Within the preceding approach, the difference in vibrational energy between $\beta$ and martensitic phases is given by

$$
\Delta E_{v i b}=\int_{0}^{T_{M}}\left(R_{C}-1\right) D d T .
$$

Therefore, the difference in configurational energy between both $\beta$ and martensite is given by

$$
\Delta E_{\text {conf }} \simeq T_{M} \Delta S-\Delta E_{\text {vib }},
$$

where $T_{M} \Delta S$ is the total-energy difference between the two phases.

\section{EXPERIMENT}

We have studied two shape-memory alloy families, $\mathrm{Cu}_{1-x} \mathrm{Zn}_{x}$ and $\mathrm{Cu}_{1-(x+y)} \mathrm{Zn}_{x} \mathrm{Al}_{y}$, with constant valence electron concentration $e / a=1+x+2 y=1.48(\mathrm{Cu}$ contributes 1 electron, $\mathrm{Zn} 2$ electrons and Al 3 electrons), which corresponds to the eutectoid value. Single crystals have been grown in sealed quartz tubes by the Bridgman method. The purity of the metals used was $99.999 \%$ in all cases. In the case of $\mathrm{Cu}-\mathrm{Zn}$ samples, after the growth process, the encapsulated ingots were kept in contact with a piece of steel at $1100 \mathrm{~K}$ while being taken out of the furnace, and rapidly quenched into water at room temperature by breaking the quartz tube. This method ensures that homogeneous $\beta$-phase $\mathrm{Cu}-\mathrm{Zn}$ single crystals are obtained. For this reason the quenched $\mathrm{Cu}-\mathrm{Zn}$ single crystals were not heat treated further except for long-term annealing at room temperature. From the ingots of both $\mathrm{Cu}-\mathrm{Zn}$ and $\mathrm{Cu}-\mathrm{Zn}-\mathrm{Al}$, small 5-mmdiameter disks of $2 \mathrm{~mm}$ thickness were cut using a low-speed diamond saw. In order to eliminate any surface damage, samples were polished with fine grinding paper. The actual composition of the single crystals was determined by means of electron dispersion analysis of $\mathrm{x}$ rays. $\mathrm{Cu}-\mathrm{Zn}-\mathrm{Al}$ samples were annealed for $900 \mathrm{~s}$ at $1073 \mathrm{~K}$, air cooled down to room temperature and annealed at room temperature for a very long time (several days).

Transition temperatures $T_{M}$, entropy changes $\Delta S$, and enthalpy changes $\Delta H$ were obtained by means of calorimetric measurements. A high-sensitivity differential scanning

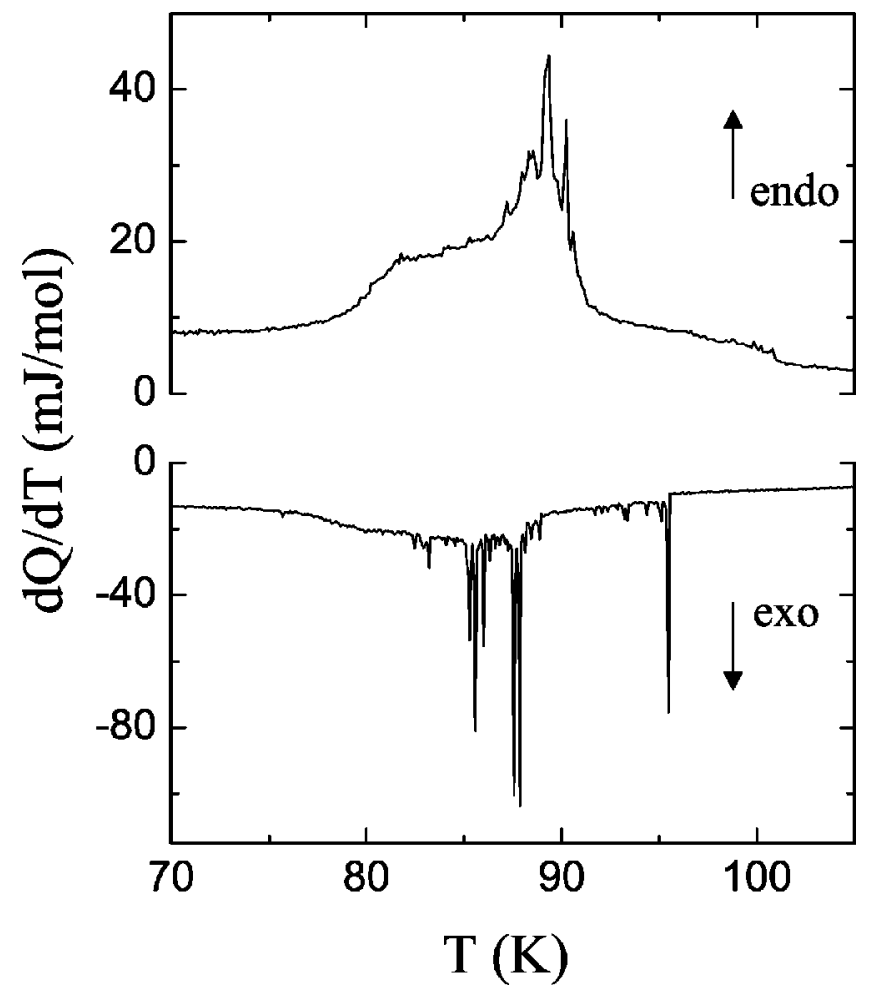

FIG. 2. Example of a calorimetric curve for a $\mathrm{Cu}_{62.58} \mathrm{Zn}_{26.91} \mathrm{Al}_{9.49}$ alloy.

microcalorimeter, specifically designed to investigate solidsolid transitions was used. The working temperature range was from $5 \mathrm{~K}$ to $350 \mathrm{~K}$, which is adequate for the study of martensitic transitions with $T_{M}$ down to $\sim 20 \mathrm{~K}$. An example of a calorimetric curve, represented as the ratio of the ther-

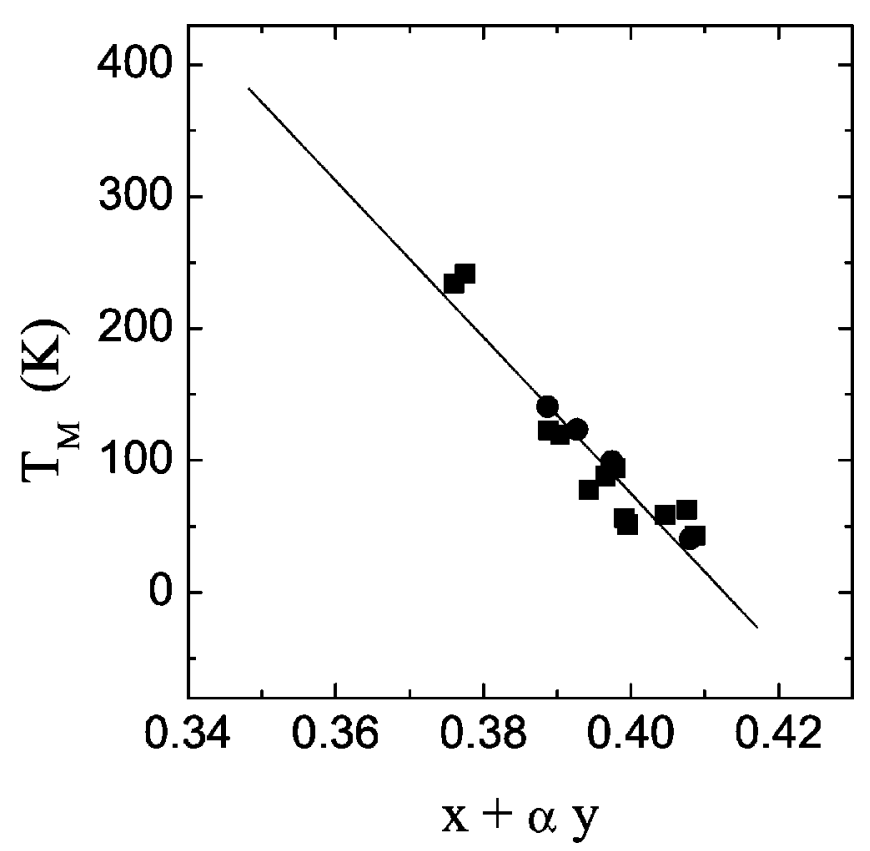

FIG. 3. Transition temperature $T_{M}$ vs reduced composition (see text for details) $x+\alpha y$ with $\alpha=1.357$ for the studied alloys. Circles: Cu-Zn. Squares: Cu-Zn-Al. 

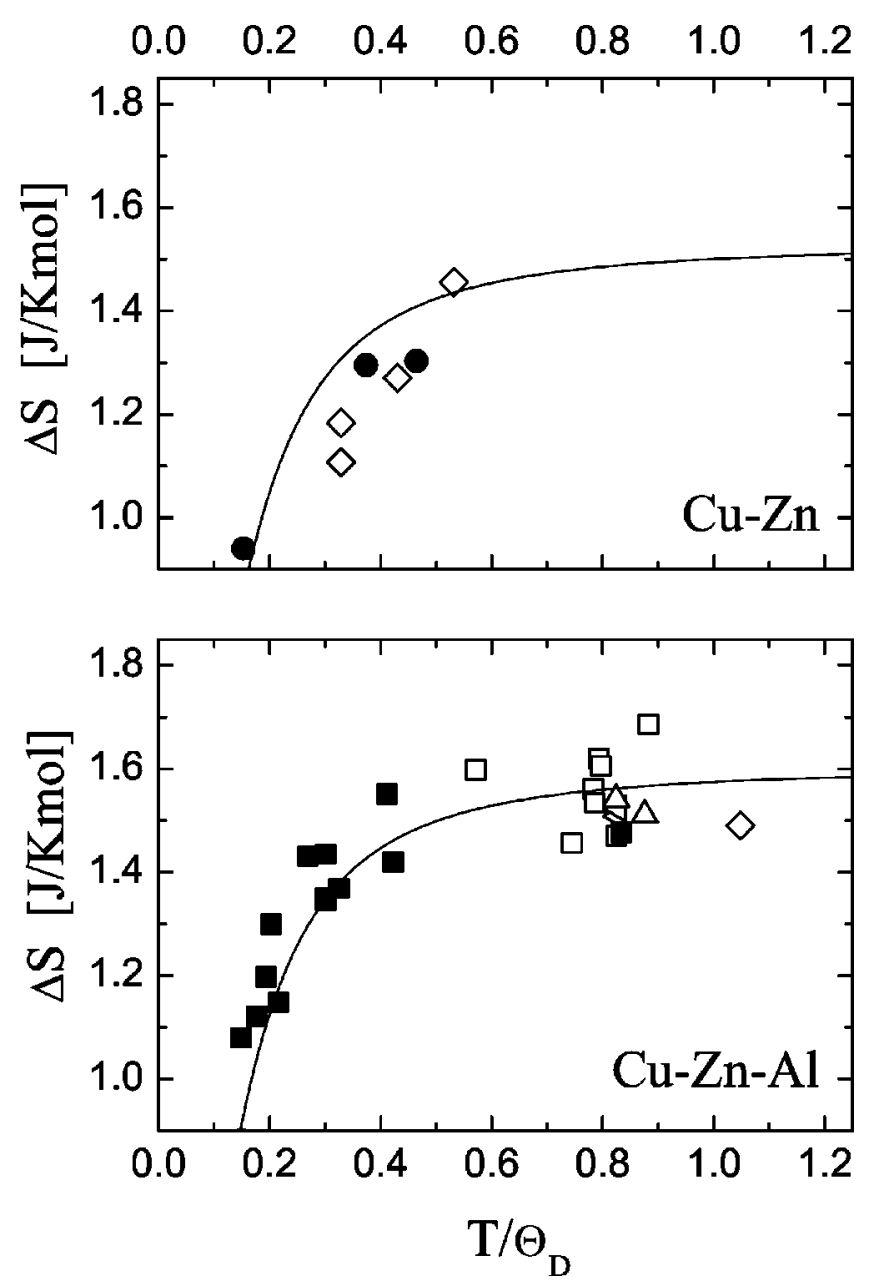

FIG. 4. Entropy change $\Delta S$ vs reduced transition temperature $T / \theta_{D}$ for $\mathrm{Cu}-\mathrm{Zn}$ and $\mathrm{Cu}-\mathrm{Zn}-\mathrm{Al}$ alloys. Solid symbols: data from the present paper. Open symbols: data collected from the literature. Continuous lines are obtained from Eq. (5) with $\theta_{D}=265 \mathrm{~K}, \theta_{E}$ $=53 \mathrm{~K}$, and $p=0.047$ for $\mathrm{Cu}-\mathrm{Zn}$ and $\theta_{D}=290 \mathrm{~K}, \theta_{E}=53 \mathrm{~K}$, and $p=0.047$ for $\mathrm{Cu}-\mathrm{Zn}-\mathrm{Al}$.

mal power $d Q / d t$ to the (instantaneous) temperature rate $d T / d t$ vs $T$ is shown in Fig. 2 for a $\mathrm{Cu}-\mathrm{Zn}-\mathrm{Al}$ crystal. The calorimetric curves show the jerky structure that is common to martensitic transitions in shape-memory alloys. After a proper correction of the base line, the enthalpy, and entropy changes can be obtained as:

$$
\Delta H=\int_{T_{i}}^{T_{f}}\left(\frac{d Q}{d t}\right)\left(\frac{d T}{d t}\right)^{-1} d T
$$

and

$$
\Delta S=\int_{T_{i}}^{T_{f}} \frac{1}{T}\left(\frac{d Q}{d t}\right)\left(\frac{d T}{d t}\right)^{-1} d T
$$

where $T_{i}$ and $T_{f}$ are temperatures located above (below) and below (above) the starting and finishing temperatures on cooling (heating) calorimetric runs, respectively.

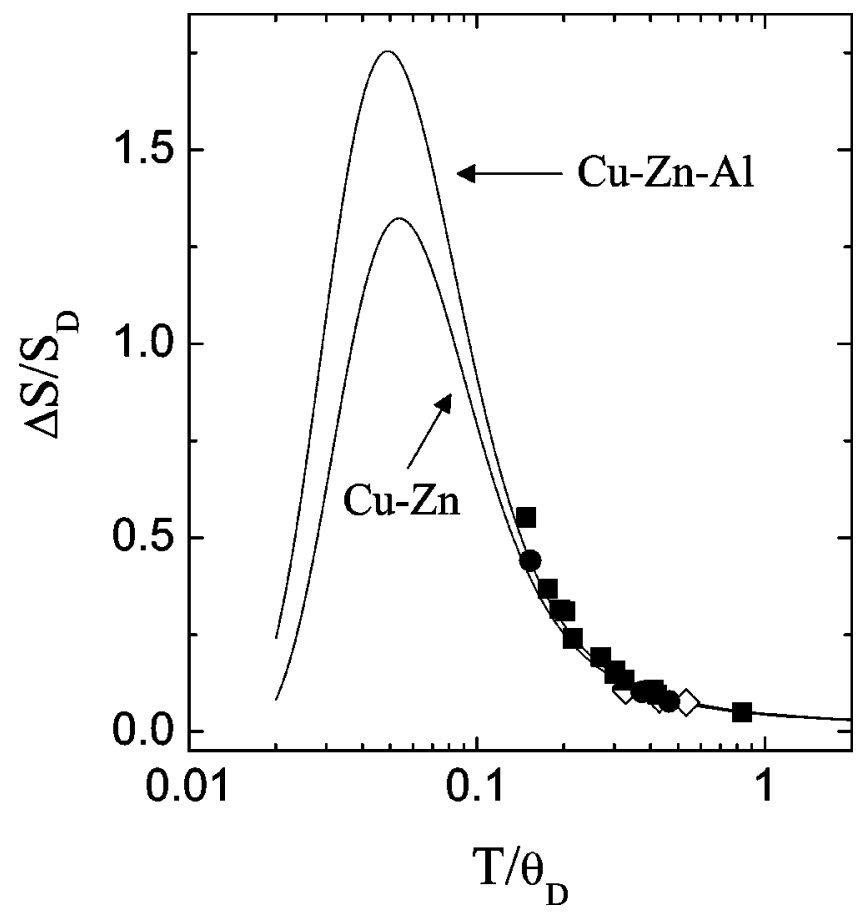

FIG. 5. Boson peak $\left(R_{S} \equiv \Delta S / S_{D}-1\right.$ vs reduced temperature $\left.T / \theta_{D}\right)$ as revealed from the difference of entropy between $\beta$ and martensitic phases. Circles: $\mathrm{Cu}-\mathrm{Zn}$. Squares: $\mathrm{Cu}-\mathrm{Zn}-\mathrm{Al}$. Solid symbols: present paper. Empty symbols: data from the literature. The continuous lines are fits to Eq. (6) with $\theta_{D}=265 \mathrm{~K}, \theta_{E}=53 \mathrm{~K}$, and $p=0.047$ for $\mathrm{Cu}-\mathrm{Zn}$, and $\theta_{D}=290 \mathrm{~K}, \theta_{E}=53 \mathrm{~K}$, and $p=0.047$ for $\mathrm{Cu}-\mathrm{Zn}-\mathrm{Al}$.

\section{RESULTS}

Figure 3 shows the transition temperature $T_{M}$ as a function of composition. For data reduction a weighted composition $x+\alpha y$ was used. It is seen that the value giving the best linear representation is $\alpha=1.357$. Actually, this value is lower than but close to, the value proposed in Ref. 12 where low-temperature transition temperatures were estimated from the resolved stress $\tau$ needed to induce the martensite at $80 \mathrm{~K}$. This estimation assumes a constant value of $d \tau / d T$ which, from the Clausius-Clapeyron equation, implies a constant value of $\Delta S$. As we will see, this is not a good assumption at low temperature and, therefore, the method supposes an underestimation of $T_{M}$. This is in agreement with the larger value of $\alpha$ reported in that work compared with the present value.

Figure 4 shows the obtained values of $\Delta S$ as a function of $T_{M}$ for $\mathrm{Cu}-\mathrm{Zn}$ and $\mathrm{Cu}-\mathrm{Al}-\mathrm{Zn}$ alloys. To our knowledge present calorimetric measurements are the only direct measurements of entropy (and enthalpy changes) for shapememory alloys which transform below liquid nitrogen. A number of data corresponding to alloys with higher $T_{M}$ have been collected from the literature and were included in the plots (open symbols). Since it is known that in Cu-based alloys the electronic contribution to the entropy change is negligibly small, ${ }^{13}$ it is thus reasonable to assume that the measured entropy difference has a purely vibrational origin. At high temperatures the entropy change is almost independent of temperature, and it decreases as the transition tem- 

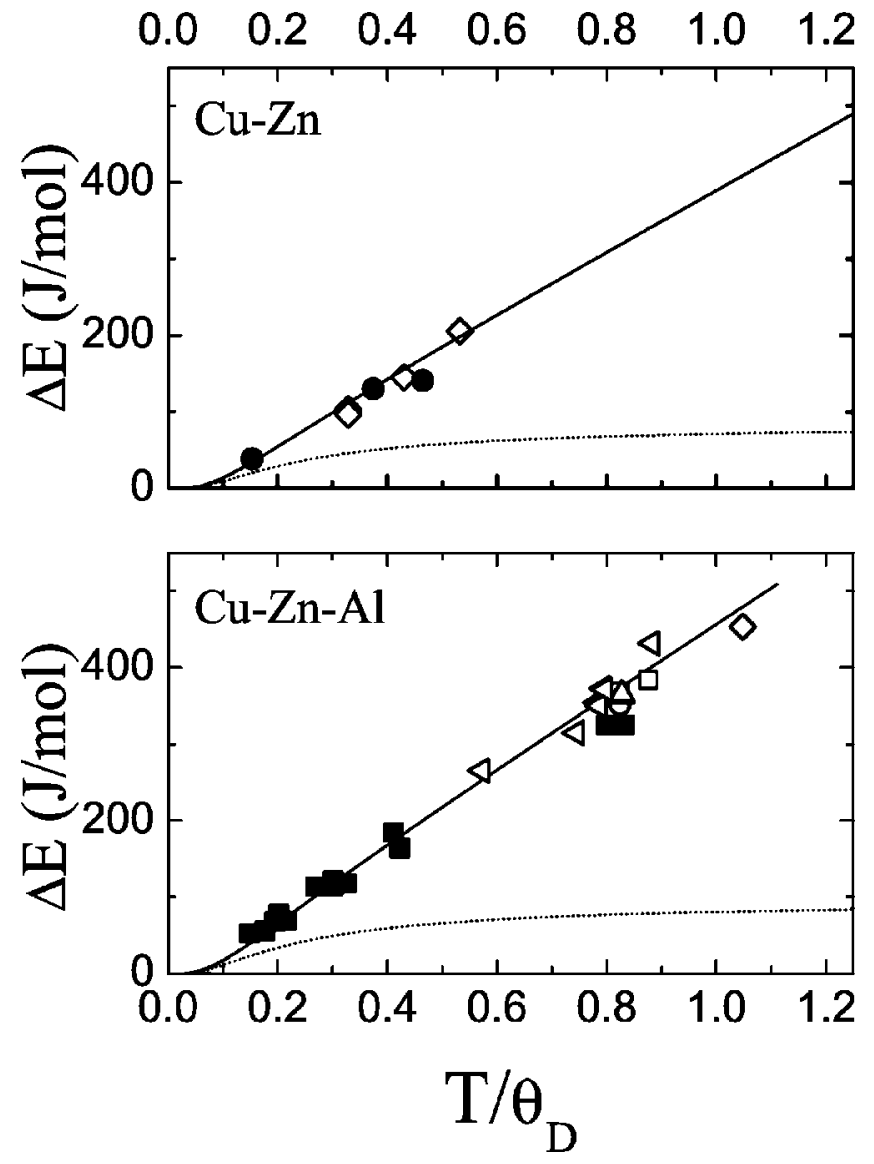

FIG. 6. Energy difference $\Delta E$ as a function of reduced transition temperature $T / \theta_{D}$. Solid symbols: this paper. Empty symbols: data from the literature. The continuous line is $T_{M} \Delta S$ and the dashed line the difference in vibrational energy $\left(\Delta E_{v i b}\right)$ obtained from the model with $\theta_{D}=265 \mathrm{~K}$ and $p=0.047$ for $\mathrm{Cu}-\mathrm{Zn}$, and $\theta_{D}=290 \mathrm{~K}$ and $p=0.047$ for $\mathrm{Cu}-\mathrm{Zn}$-Al. For both alloys the fit renders $\theta_{E}$ $=53 \mathrm{~K}$.

perature lowers. Such a decrease is in agreement with the third law of thermodynamics.

In order to compare the measured $\Delta S$ values with the predictions of the above-presented model, we need estimations of the parameters $\theta_{D}, p$, and $\theta_{E}$. We started by taking the values of $p$ and $\theta_{D}$ reported in previous works. For $\mathrm{Cu}-\mathrm{Zn}$ the Debye temperature was obtained by Veal and Rayne $^{14}$ from very low-temperature heat-capacity measurements. An average value for the compositions studied in the present paper is $\theta_{D}=265 \mathrm{~K}$. For $\mathrm{Cu}-\mathrm{Zn}-\mathrm{Al}$ alloys, $\theta_{D}$ was estimated in Ref. 15. For the composition range of interest here we take an average value of $\theta_{D}=290 \mathrm{~K}$.

On the other hand, $p$ was also estimated in Ref. 15 by fitting $\Delta S$ values of alloys transforming at high temperatures to expression (7) taking $\theta_{E}=\hbar \omega_{Z B} / k \simeq 58 \mathrm{~K}$, where $\omega_{Z B}$ is the zone-boundary frequency of the $T A_{2}$ branch measured at a temperature close to the martensitic transition temperature. These fits yield a value of $p \simeq 0.047$, which was found to be nearly independent of alloy composition. We thus fixed this value of $p$ for both $\mathrm{Cu}-\mathrm{Zn}$ and $\mathrm{Cu}-\mathrm{Zn}-\mathrm{Al}$ alloys. As regards $\theta_{E}$, the estimation based on the $T A_{2}$ boundary frequency $\omega_{Z B}$ certainly represents an overestimation. Therefore, this parameter will be left as a free fitting parameter.

We fitted expression (6) to the ratio between the measured $\Delta S$ and $S_{D} \cdot S_{D}$ and $S_{\beta}$ were determined by numerical integration. Best fits were obtained with $\theta_{E}=53 \mathrm{~K}$ for both $\mathrm{Cu}-\mathrm{Zn}$ and $\mathrm{Cu}-\mathrm{Zn}-\mathrm{Al}$. As expected, these values are slightly lower than previous estimations based on the zone-boundary frequency. The fits are depicted in Fig. 5, where $R_{S}-1$ $\left(=\Delta S / S_{D}\right)$ vs $T_{M} / \theta_{D}$ is plotted for $\mathrm{Cu}-\mathrm{Zn}$ and $\mathrm{Cu}-\mathrm{Zn}-\mathrm{Al}$ alloys, respectively. The experimental results clearly show the tendency expected for a system displaying the BP. The continuous lines in Fig. 4 are obtained from Eq. (5) with the values estimated for the parameters $\theta_{D}, \theta_{E}$, and $p$. The goodness of the fit gives further support to the existence of the BP.

Since the volume change at the martensitic transition in $\mathrm{Cu}$-based shape-memory alloys is negligibly small, the calorimetrically measured $\Delta H$ is an excellent estimation of the energy difference between the two phases. In Fig. 6 the measured values of $\Delta E$ are compared with $T_{M} \Delta S$, where $\Delta S$ is obtained with the values of $p, \theta_{D}$, and $\theta_{E}$ previously determined. The agreement is very satisfactory. $\Delta E_{v i b}$ calculated from Eq. (10) is also shown in the figure. This quantity is small, but plays an important role in the low-temperature transition region. For alloys transforming at temperatures $T_{M}>\theta_{E}$, the energy difference is essentially determined by the configurational energy difference and shows a linear dependence on the transition temperature.

\section{DISCUSSION}

In this paper we have reported low-temperature measurements of the energy and entropy differences between $\beta$ and martensitic phases in $\mathrm{Cu}-\mathrm{Zn}$ and $\mathrm{Cu}-\mathrm{Zn}-\mathrm{Al}$ with an eutectoid composition. We have confirmed that the excess of entropy of the $\beta$ phase with respect to the martensitic phases arises from the low-energy $T A_{2}$ phonon modes. These low-energy modes show up in the density of states as a BP with similar features as the BP reported for a number of disordered solids. This anomaly is observed about frequencies close to the zone-boundary frequency of the $T A_{2}$ branch. This behavior has been accounted for by assuming a DOS with a certain fraction $p$ of localized modes described by an Einstein model and the remaining modes described by a Debye model. The DOS of the close-packed martensite is assumed to be Debye type with the same Debye temperature as in the corresponding $\beta$ phase. We have found that this simple model provides excellent results regarding thermodynamic quantities such as the entropy difference between the two phases. In particular, the model enables understanding of why the entropy change takes a rather constant value over a broad temperature range (see Fig. 4): from high temperature down to temperatures slightly above the Einstein temperature which characterizes the anomalous low-energy modes. Moreover, the small contribution of the vibrational energy to the energy difference between the two phases is also explained by the model taking into account the small value of the fraction $p$ of these anomalous modes.

The three relevant parameters of the proposed model are the Einstein $\theta_{E}$ and the Debye $\theta_{D}$ temperatures and the fraction $p$ of anomalous modes. $\theta_{E}$ and $p$ are seen to be, to a 
good approximation, composition independent, while $\theta_{D}$ depends on composition essentially through the average atomic mass. It is interesting to analyze Eq. (9) with the estimated values of these parameters, together with the measured value of the entropy change at high temperatures. Assuming that $T_{M} \simeq 300 \mathrm{~K}$, the mean amplitude displacements of (110) planes along [1 $\overline{1} 0]$ direction that enable the instability of the $\beta$ phase to yield the martensitic structure are of the order of $10 \%$ of the interplanar distance between (110) planes. Actually, this is a reasonable value for $\mathrm{Cu}$-based shape-memory alloys taking into account the fact that the transformation strain is of the order of $15 \%$.

At a first glance, the existence of the BP in shape-memory alloys could be considered as an unexpected result since this feature is commonly associated with structural disorder. Actually, in disordered solids its appearance is still a subject of speculation and different explanations have been proposed. ${ }^{16}$ It seems, however, that disorder-induced localization of crystal vibrations is at the origin of such an effect. Since disorder is not the only possible localization effect, this point of view enables us to propose that the BP should be present in any system with localized vibrational modes. This is, for instance, supported by recent inelastic neutron scattering measurements in Tl-filled skutterudites. ${ }^{17}$ Results show a peak in the DOS, absent in the unfilled system, which is unambigu- ously attributed to localized modes associated with the $\mathrm{Tl}$ atoms. The results of the present paper are consistent with this viewpoint. In shape-memory systems, the strong anisotropy of the open $\beta$ phase, characteristic of these systems, ${ }^{5}$ causes the localization of the soft anomalous (110)[1 $\overline{1} 0]$ modes. This is in agreement with very general arguments claiming that strong anisotropy and/or noncentral forces are factors that favor the existence of low-energy modes giving rise to the $\mathrm{BP}^{18}$ In the close-packed martensitic phase with low elastic anisotropy the anomaly disappears and the material displays, to a large extent, the usual Debye behavior. It is foreseeable that the observed behavior could also occur in other crystalline solids with low-lying soft branches such as certain ferroelectrics.

\section{ACKNOWLEDGMENTS}

We are grateful to Professor Jim Krumhansl for calling our attention to the possibility of finding a boson peak in shape-memory alloys. We thank Avadh Saxena for fruitful discussions. This work has received financial support from CICyT (Project No. MAT2001-3251) and DURSI (Project No. 2001SGR00066). J.M. acknowledges financial support from DURSI.
*Permanent address: IFIMAT, Universidad del Centro de la Provincia de Buenos Aires, Pinto 399, 7000 Tandil, Argentina.

${ }^{1}$ C. Zener, Phys. Rev. 71, 846 (1947).

${ }^{2}$ A. Zangwill and R. Bruinsma, Comments Condens. Matter Phys. 13, 1 (1987).

${ }^{3}\{110\}$ planes of the $\beta$-phase transform to $\{100\}$ planes in the martensite. Therefore, the $T A_{2}$ branch of the parent becomes the $T A_{1}$ branch in the martensitic phase.

${ }^{4}$ G. Guénin, R. Pynn, D. Ríos-Jara, L. Delaey, and P.F. Gobin, Phys. Status Solidi A 59, 553 (1980).

${ }^{5}$ A. Planes and Ll. Mañosa, Solid State Phys. 55, 159 (2001).

${ }^{6}$ B. Frick and D. Richter, Science 267, 1939 (1995).

${ }^{7}$ W. Schirmacher, G. Diezemann, and C. Ganter, Phys. Rev. Lett. 81, 136 (1998).

${ }^{8}$ F.C. Lovey, Acta Metall. 35, 1103 (1987).

${ }^{9}$ G. Gilat and G. Dolling, Phys. Rev. 138, A1053 (1965).
${ }^{10}$ L1. Mañosa, J. Zarestky, M. Bullock, and C. Stassis, Phys. Rev. B 59, 9239 (1999).

${ }^{11}$ K. Nicolaus, Ph.D. thesis, Technishe Universität München, 2000 (unpublished).

${ }^{12}$ A. Planes, R. Romero, and M. Ahlers, Acta Metall. Mater. 38, 757 (1990).

${ }^{13}$ L1. Mañosa, A. Planes, J. Ortín, and B. Martínez, Phys. Rev. B 48, 3611 (1993).

${ }^{14}$ B.W. Veal and J.A. Rayne, Phys. Rev. 128, 551 (1962).

${ }^{15}$ R. Romero and J.L. Pelegrina, Mater. Sci. Eng., A 354, 243 (2003).

${ }^{16}$ V.L. Gurevich, D.A. Parshin, and H.R. Schober, Phys. Rev. B 67, 094203 (2003).

${ }^{17}$ R.P. Hermann, R. Jin, W. Schweika, F. Grandjean, D. Mandrus, B.C. Sales, and G.J. Long, Phys. Rev. Lett. 90, 135505 (2003).

${ }^{18}$ J.A. Krumhansl, Phys. Rev. Lett. 56, 2696 (1986). 